\title{
Density-functional calculations of carbon doping in III-V compound semiconductors
}

\author{
C. D. Latham, ${ }^{1, *}$ R. Jones, ${ }^{1}$ S. Öberg, ${ }^{2}$ and P. R. Briddon ${ }^{3}$ \\ ${ }^{1}$ School of Physics, University of Exeter, Exeter, EX4 4QL, United Kingdom \\ ${ }^{2}$ Department of Mathematics, Luleå University of Technology, SE-97187 Luleå, Sweden \\ ${ }^{3}$ Department of Physics, University of Newcastle upon Tyne, Newcastle, NE1 7RU, United Kingdom
}

(Received 17 July 2000; published 29 March 2001)

\begin{abstract}
This article reports the results of investigations based on local-density-functional theory into the relative formation energies for single substitutional carbon atoms in nine III-V compound semiconductors. The calculations are performed using a supercell formalism derived from the AIMPRO real-space cluster method. Only a very slight trend is discernible down the periodic table. When a metal atom is replaced with carbon, it is energetically least favorable in the phosphides, very marginally lower energy in the arsenides, and $\approx 0.5-0.7$ $\mathrm{eV}$ lower in the antimonides. The situation is approximately reversed when a $\mathrm{P}, \mathrm{As}$, or $\mathrm{Sb}$ atom is substituted by a $\mathrm{C}$ atom: for the In compounds the energy is $\approx 0.4-0.8 \mathrm{eV}$ higher than for the $\mathrm{Al}$ and $\mathrm{Ga}$ compounds.
\end{abstract}

DOI: 10.1103/PhysRevB.63.155202

PACS number(s): 61.72.Bb, 61.72.Ji, 61.72.Vv, 71.15.Nc

\section{INTRODUCTION}

Carbon has been found to be a convienient dopant for III-V semiconductor crystals for a variety of reasons. In many cases, for example, it is already included in the precursors used to make a given material, and this can allow very high concentrations to be achieved. Another of its advantages is that the bonds it forms with the atoms of most hosts are strong compared with those of other dopant atoms, and therefore the diffusion rate is normally low. This means that it is possible to maintain sharp boundaries to doped regions during processing and operation of devices. Inevitably, there are limitations to using carbon as a dopant. Perhaps the most obvious of these is that, in general, carbon atoms may act as donors or acceptors depending on whether they replace group-III or group-V atoms in a crystal. The ratio of carbon atoms occupying one site relative to the other in different specimens has been observed to vary over nearly the full range of values from one extreme to the other. This has a direct effect on the net fraction of majority carriers with respect to the concentration of dopant atoms. The latter quantity is also known as the doping efficiency, with the implication that the closer the figure is to $100 \%$, the better it is.

The site occupancy ratio is not the only factor controlling doping efficiency. As the concentration of dopant atoms increases, the chemical potential tends to drive the formation of other compensating defects. In the first instance, however, it is expected that the relative strengths of the bonds carbon atoms form with those of the host they reside in will affect the outcome. This is one of the first points made in a general review covering many aspects of carbon doping in III-V semiconductors by Abernathy. ${ }^{1}$ The article shows, among other things, how the maximum observed carrier concentration and majority carrier type in these materials is related to the differences in bond strengths between the host and carbon atoms. Although there are some gaps and uncertainties in the experimental data, the trends with elemental type appear to be broadly in line with expectations. For example, $\mathrm{GaAs}, \mathrm{GaSb}$, and AlAs are reported as being strongly $p$ type (maximum observed $[p] \geq 10^{20} \mathrm{~cm}^{-3}$ ) and metal-carbon bonds are stronger than $\mathrm{C}$-As and $\mathrm{C}-\mathrm{Sb}$ bonds. This relation- ship between bond strengths and majority carrier type is also found in earlier work by Ito and Ishibashi. ${ }^{2}$ They measured the electronic properties of the Al-Ga-In-As system of ternary alloys for the whole range of mole fractions. A transition from $p$ - to $n$-type electrical conduction was observed when the InAs mole fraction for $\mathrm{Ga}_{1-x} \mathrm{In}_{x}$ As exceeded 0.6, and 0.9 for $\mathrm{Al}_{1-x} \mathrm{In}_{x}$ As.

Growth conditions and processing following growth are two other crucial factors that also have a strong influence on the observed majority carrier concentration, Abernathy notes. During growth, competition for sites in a crystal can be altered by changing the ratios of the precursors, or by choice of crystallographic orientation, although this turns out to be a complex issue and is not well understood. InP is a case in point: Abernathy reports it as being compensated $n$ type $\left([\mathrm{n}] \sim 6 \times 10^{19} \mathrm{~cm}^{-3}\right)$; however, Newman et al. note that the expected high-frequency local vibrational modes of $\mathrm{C}_{\mathrm{In}}$ donors have not conclusively been observed. ${ }^{3}$ Moreover, Davidson, Newman, and Button have detected $C_{P}$ acceptors and $\mathrm{C}_{\mathrm{P}}-\mathrm{H}$ pairs in semi-insulating material. ${ }^{4}$ All of the carbon in their material $\left([\mathrm{C}] \sim 3 \times 10^{18} \mathrm{~cm}^{-3}\right)$ was accounted for by these two types of center in approximately equal amounts. The nature of the compensating defect or defects is unknown, but could be $\mathrm{P}_{\text {In }}$ double-donor antisite defects or hydrogenated vacancy $\left(V \mathrm{H}_{4}\right)$, shallow donors. Dicarbon defects, $(\mathrm{C}-\mathrm{C})_{\mathrm{P}}$, similar to those observed in AlAs and GaAs, may also be responsible. ${ }^{5-7}$ These are deep donors, and we understand there is spectroscopic evidence for them. ${ }^{8}$ An interpretation of this unusual behavior could be that the creation of $\mathrm{C}_{\mathrm{P}}$ acceptors is always accompanied by the formation of a greater or equal number of donors, in a similar manner to the codoping technique, which enhances electrical activity of one type by the deliberate addition of the other type for reasons which are not understood.

The main aim of this article is to concentrate on providing theoretical estimates of relative formation energies for single substitutional carbon atoms in nine III-V semiconductors in order to try and discern more clearly the trends if any, down these two columns of the periodic table. This is not a new idea: previously Weyers and Shiraishi calculated energies for placing a single, isolated carbon atom into a vacancy in the 
phosphides and arsenides of $\mathrm{Al}, \mathrm{Ga}$, and In. ${ }^{9}$ They used a local-density-functional, hard-core pseudopotential method with a fully plane-wave basis, and a fixed $X_{\alpha}$ potential for the exchange-correlation potential. The authors concluded that, except for InAs, their calculations gave results generally in agreement with the experimentally observed chemical trends, for both the uptake of carbon and the resulting electronic properties then known. However, the scope and accuracy of the calculations was compromised by the resources available at the time (1992). Supercells containing eight atoms were used for the main calculations, and relaxation of the atomic coordinates was restricted to nearest neighbors only. Weyers and Shiraishi reported that, for a sample pair of calculations using 64-atom supercells, the energies for the two vacancies in GaAs were different by $0.25 \mathrm{eV}$ compared with the eight-atom supercells. As will be shown next, it is now possible to perform calculations free from such restrictions.

\section{METHOD}

A short description of the AIMPRO supercell method is given in a recent article by Coutinho et al. ${ }^{10}$ and further details are provided in Ref. 11. Essentially, it is a method based on self-consistent total energy local-density-functional theory. It uses the spin-averaged exchange-correlation energy functional parametrized by Perdew and Wang, ${ }^{12}$ together with Bachelet-Hamann-Schlüter pseudopotentials ${ }^{13}$ replacing the core electrons of atoms. The Kohn-Sham wave functions of the valence electrons are expanded in basis sets of Gaussian orbitals. These have $s$ - and $p$-orbital symmetries provided by suitable multiplicative factors. The charge density is represented by a plane-wave basis in reciprocal space, together with a Monkhorst-Pack (MP) scheme ${ }^{14}$ to sample the band structure. Both the largest reciprocal lattice vector of the charge-density Fourier expansion, and the mesh of $\mathbf{k}$ points are chosen so that the total energy $E_{\text {total }}$ is converged to better than the required limit with respect to these parameters. An automatic procedure ensures that the number of shells of vectors $\mathbf{R}_{\mathbf{L}}$ used to evaluate the Madelung energy is sufficient. The forces acting on each atom are given by an analytical formula derived from the total energy expression. Structural optimization to minimize the total energy is performed by a conjugate-gradient algorithm. The lattice vectors describing a supercell are held fixed during the energy minimization procedure. In the case of cubic supercells, the lattice parameter $a_{0}$ can be optimized separately by calculating the minimum total energy for several (minimum 5) values of $a_{0}$ over a range of $1-3 \%$, and fitting the points to the Birch-Murnaghan equation of state. An additional energy point is then calculated at the estimated equilibrium value of $a_{0}$ to check the result. This procedure naturally also yields a value for the bulk modulus.

The wave function basis sets used to obtain the results presented here consisted of four Gaussian-type orbitals in $s, \quad p_{x}, p_{y}, p_{z}$ variants, with different, fixed widths, but variable amplitude, centered on each host and carbondopant atom. Supplementary Gaussian-type basis functions were placed at the center of every bond. Experience gained from the earlier work by Coutinho et al. ${ }^{10}$ showed that the best basis sets generated using a Monte Carlo algorithm differed very little from geometric series; hence it was decided that even-tempered sets should be used as this would simplify matters and probably make little difference. Optimum values of the Gaussian exponents were then determined by conducting an exhaustive search of all possible combinations over atom types and bond centers, and values for the lower and upper exponents, which themselves were generated in geometric series over a range. The ranges of values considered for lower and upper exponents were selected partly by what were believed to be "reasonable values" and partly by trial and error. Typically, 20000-30000 unique sets were tested for each of 13 of the 16 possible combinations of the elements $\mathrm{B}, \mathrm{Al}, \mathrm{Ga}$, and In with $\mathrm{N}, \mathrm{P}, \mathrm{As}$, and $\mathrm{Sb}$ in twoatom primitive unit cells in an fcc lattice, and with the the lattice parameter $a_{0}$ fixed at the measured values. The three not included were BP, BAs, and BSb. Basis sets for $\mathrm{C}, \mathrm{Si}$, $\mathrm{Ge}$, and $\mathrm{SiC}$ in the diamond structure were also generated in a similar manner.

A few basis sets tried yielded results that were clearly spurious, i.e., the total energy was well outside what could safely be considered reasonable, or resulted in failure of the computer program and hence could be rejected. The remaining ones were found to give total energies that lay in a narrow band of values $(\sim 1 \mathrm{eV} / \mathrm{atom})$ for a given material. Of these, the basis sets giving the lowest total energy in each material were selected as the "best" candidates. It was found that in general for a given type of atom in each material the atom-centered exponents tended to be similar, but that the width of the bond-centered exponents varied slightly, such that crystals with larger values for $a_{0}$ had broader Gaussians. Subsequent testing showed that the basis sets were all reasonably transferable. In other words, if the optimium atom basis for one material was used in another from the set previously described, the total energy per atom increased by typically $\lesssim 0.01 \mathrm{eV}$. Taken together, these results indicate that the basis sets are to some extent overcomplete, thus validating the decision to restrict the exponent values to geometric series. Moreover, the basis sets found for C (diamond), $\mathrm{Si}$, and $\mathrm{Ge}$ by this method yielded lower energies per atom by $0.16,0.01$, and $0.05 \mathrm{eV}$, respectively, than those generated previously by Coutinho et al. ${ }^{10}$

\section{RESULTS}

\section{A. Ideal crystals}

The equilibrium values of $a_{0}$ and the bulk modulus were calculated for all possible combinations of elements being considered here (including diamond, silicon, germanium, and silicon carbide), and over all of the optimized basis sets for the ideal cubic zinc-blende structure in two-atom primitive unit cells on an fcc lattice. Tests showed that the total energy $E_{\text {total }}$ was fully converged for all the calculations using a mesh of 28 special $\mathbf{k}$ points sampling the irreducible wedge of the Brillouin zone (MP $6^{3}$ scheme), and a cutoff at 600.0 Ry in the charge-density Fourier expansion. In every case the particular combination of basis sets made no significant dif- 
TABLE I. Calculated and measured (Refs. 18 and 19) lattice parameters for cubic structure III-V semiconductors $(\AA)$.

\begin{tabular}{lccc}
\hline \hline & Calculated & Measured & Ratio $(\%)$ \\
\hline $\mathrm{AlP}$ & 5.4317 & 5.4635 & 99.4 \\
$\mathrm{AlAs}$ & 5.6196 & 5.6605 & 99.3 \\
$\mathrm{AlSb}$ & 6.0906 & 6.1355 & 99.3 \\
$\mathrm{GaP}$ & 5.3153 & 5.4505 & 97.5 \\
$\mathrm{GaAs}$ & 5.5140 & 5.6533 & 97.5 \\
$\mathrm{GaSb}$ & 5.9610 & 6.0959 & 97.8 \\
$\mathrm{InP}$ & 5.6750 & 5.8688 & 96.7 \\
$\mathrm{InAs}$ & 5.8617 & 6.0584 & 96.8 \\
$\mathrm{InSb}$ & 6.2981 & 6.4794 & 97.2 \\
\hline \hline
\end{tabular}

ference to the results. Except for $\mathrm{GaN}$ and $\mathrm{InN}$, the lattice parameter was always slightly smaller $(0.5-3.3 \%)$ than the measured value: this is a normal consequence of the pseudopotential approximation. Where reliable comparisons could be made, the bulk modulus was found to be within $5 \%$ of the measured value.

It was decided at this stage to exclude the compounds of boron and nitrogen from the carbon-doping calculations for several reasons. First, none of the existing boron compounds have significant electronic application at present. However, for the record, the $\mathrm{B}$ and $\mathrm{N}$ basis sets yielded a value for $a_{0}$ in $c$-BN that was $98.9 \%$ of the measured one. Cubic AlN was also a success (calc. $a_{0}=98.4 \%$ of the true value). The main problem lies with $\mathrm{GaN}$ and InN. Unfortunately, the theoretical method used here performs very poorly for these two nitrides. This is due to there being a resonance between the $3 d$ core electrons in $\mathrm{Ga}$, or $4 d$ core electrons in In, and the $2 p$ valence electrons in N. As described previously, the core electrons are replaced by a pseudopotential; hence these interactions in $\mathrm{GaN}$ and $\mathrm{InN}$ are not adequately described. This problem may be addressed in the future by means of a core correction. It is not a practical proposition to explicitly include the core $d$ electrons: the computational demands would be much greater than present-day resources normally permit, and numerical instability is a problem.

\section{B. Carbon-doped crystals}

Having established basis sets and structures for the ideal crystals as given in Table I, the next step was to replace a group-III or a group-V atom in supercells of each material with a carbon atom. In all these calculations we model only an ideal single substitutional carbon atom with perfect $T_{d}$ symmetry in the crystal, and in the neutral charge state. Possible off-site distortions and other charge states are ignored in order that their effects may be kept separate.

Two sizes of supercell containing either 64 or 216 atoms were used. The purpose of this is simply to examine the effect of supercell size, and hence test whether the model is sufficiently large to describe the system properly. There are two main concerns about the size of supercell in this particular case. The first of these is that substitutional carbon atoms are in general expected to make shallow levels appear in the band gap, with the implication that the Bohr orbits of their
TABLE II. Calculated effects of allowing $a_{0}$ to relax for 64atom supercells containing a single substitutional $\mathrm{C}$ atom.

\begin{tabular}{llll}
\hline \hline & $\begin{array}{c}\mathrm{AlAs} \\
\mathrm{C}_{M}\end{array}$ & GaAs & InAs \\
& 5.5895 & 5.4899 & 5.8267 \\
Relaxed $a_{0}(\AA)$ & 0.53 & 0.44 & 0.60 \\
$-\Delta a_{0} / a_{0}(\%)$ & 0.08 & 0.05 & 0.10 \\
$-\Delta E_{\text {total }}(\mathrm{eV})$ & $\mathrm{C}_{\mathrm{As}}$ & & \\
& 5.5813 & 5.4773 & 5.8257 \\
Relaxed $a_{0}(\AA)$ & 0.68 & 0.67 & 0.61 \\
$-\Delta a_{0} / a_{0}(\%)$ & 0.14 & 0.13 & 0.11 \\
$-\Delta E_{\text {total }}(\mathrm{eV})$ & & & \\
\hline \hline
\end{tabular}

ground state wave functions are too large to be contained even within the dimensions of the 216-atom supercell used here, and will therefore overlap significantly with the image wave function in neighboring cells. Having said that, it should be noted that in some materials, notably AlAs and $\mathrm{GaAs}$, the maximum carbon concentration [C] achieved in experiments is nearly the same as $[\mathrm{C}]$ in the 64-atom supercell, $6.92 \times 10^{20} \mathrm{~cm}^{-3}$.

The second concern relates to the consequences of doping a crystal with an atom type that has a size much smaller than those of the host crystal. This results in a large strain which, if allowed to relax, yields a contraction of the lattice parmeter $a_{0}$. The magnitude of this contraction, $\Delta a_{0} / a_{0}$, may be estimated using Vegard's law. For example, in GaAs if one $\mathrm{Ga}$ atom in 64 is replaced by a $\mathrm{C}$ atom, $\Delta a_{0} / a_{0}=-0.55 \%$; and when As is replaced by carbon at this concentration, $\Delta a_{0} / a_{0}=-0.62 \%$. Clearly, if [C] is reduced to one in 216 atoms $\left(2.05 \times 10^{20} \mathrm{~cm}^{-3}\right)$ any effects due to contraction of the lattice or strain will be smaller. The corresponding figures given by Vegard's law for $\Delta a_{0} / a_{0}$ in GaAs at this concentration now become $-0.18 \%$ and $-0.16 \%$ when $\mathrm{C}$ occupies $\mathrm{Ga}$ and As sites, respectively.

In the next set of results shown in Table II, 64-atom supercells of AlAs, GaAs, and InAs each containing a single substitutional carbon atom replacing either the metal or the As atom were relaxed, and the equilibrium lattice parameter was determined by the procedure described previously. All the calculations using a 64-atom supercell required a sampling mesh of four special $\mathbf{k}$ points (MP $4^{3}$ scheme), and a cutoff of 160.0 Ry for the charge-density Fourier expansion to achieve convergence in the total energy to better than $0.003 \mathrm{eV}$.

In all three examples the effects of carbon doping are clearly significant. The shrinkage in lattice parameter, $\Delta a_{0} / a_{0}$, from the calculated values for $a_{0}$ given in Table I, and the decrease in total energy associated with this, $\Delta E_{\text {total }}$, are slightly more when a $\mathrm{C}$ atom replaces an As atom than when it replaces a metal atom. This is probably a consequence of $\mathrm{C}-M$ bonds $(M=\mathrm{Al}, \mathrm{Ga}, \mathrm{In})$ being stronger than C-As bonds. Note that for GaAs the values for $\Delta a_{0} / a_{0}$ calculated here agree well with the predictions of Vegard's law, which is itself well known to be a good approximation in most circumstances when compared with experimental measurements. 
TABLE III. Formation energy for a substitutional carbon atom defect relative to an arbitrary chemical potential: $E_{\text {defect }}-E_{\text {ideal }}$.

\begin{tabular}{|c|c|c|c|}
\hline \multicolumn{4}{|c|}{$\mathrm{C}_{M}(\mathrm{eV})$} \\
\hline$M$ & $M \mathrm{P}$ & $M$ As & $M \mathrm{Sb}$ \\
\hline \multicolumn{4}{|c|}{ 64-atom supercell } \\
\hline $\mathrm{Al}$ & -93.37 & -93.37 & -94.10 \\
\hline $\mathrm{Ga}$ & -89.07 & -89.11 & -89.64 \\
\hline In & -93.97 & -94.05 & -94.57 \\
\hline \multicolumn{4}{|c|}{ 216-atom supercell } \\
\hline $\mathrm{Al}$ & -93.44 & -93.42 & -94.16 \\
\hline $\mathrm{Ga}$ & -89.10 & -89.14 & -89.71 \\
\hline In & -94.05 & -94.13 & -94.66 \\
\hline \multicolumn{4}{|c|}{$\mathrm{C}_{X}(\mathrm{eV})$} \\
\hline$X$ & $\mathrm{Al} X$ & $\mathrm{GaX}$ & $\operatorname{In} X$ \\
\hline \multicolumn{4}{|c|}{ 64-atom supercell } \\
\hline $\mathrm{P}$ & 27.57 & 27.48 & 28.00 \\
\hline As & 20.42 & 20.45 & 21.00 \\
\hline $\mathrm{Sb}$ & -0.71 & -0.40 & 0.06 \\
\hline \multicolumn{4}{|c|}{ 216-atom supercell } \\
\hline $\mathrm{P}$ & 27.50 & 27.36 & 27.90 \\
\hline As & 20.31 & 20.27 & 20.86 \\
\hline $\mathrm{Sb}$ & -0.92 & -0.68 & -0.16 \\
\hline
\end{tabular}

The method used here does not work at present with metals; hence it cannot provide formation energies relative to the elements in their standard state. Although there is an option to set a finite electron temperature that occupies the KohnSham electronic states according to a Fermi distribution, and which suppresses numerical instability in the selfconsistency solution, the code fails to give a stable, converged total energy. We are therefore restricted to relative formation energies. These may be most usefully expressed in two ways: (1) relative to an arbitrary chemical potential, i.e., $E_{\text {defect }}-E_{\text {ideal }}$, or (2) relative to each other, i.e., $\left[E(b)_{\text {defect }}-E(b)_{\text {ideal }}\right]-\left[E(a)_{\text {defect }}-E(a)_{\text {ideal }}\right]$. The second of these represents the energy to swap a single substitutional carbon atom contained in a host crystal $(a)$ with an atom of the type it replaces from a different, undoped crystal (b), leaving the first crystal in the ideal, undoped state. For example, if $a$ is AlP and $b$ is GaP, this is the energy of $\mathrm{C}_{\mathrm{P}}$ in $\mathrm{GaP}$ relative to $\mathrm{C}_{\mathrm{P}}$ in $\mathrm{AlP}$.

Supercells containing 64 atoms of the remaining compounds (AlP, GaP, InP, AlSb, GaSb, and InSb), each containing a single substitutional carbon donor or acceptor in the neutral state, were next relaxed holding $a_{0}$ at the values determined for the ideal crystals, as given in Table I. Finally, the whole set of calculations at fixed $a_{0}$ were repeated using supercells containing 216 atoms. As in the 64-atom supercells, a sampling mesh of four special $\mathbf{k}$ points (MP $4^{3}$ scheme) and a cutoff of $160.0 \mathrm{Ry}$ were needed to achieve convergence in the total energy to better than $0.003 \mathrm{eV}$. The relative formation energies for carbon donors and acceptors calculated in this way are shown in Tables III and IV.

It is immediately clear that the results for the 64-atom supercells and the 216-atom ones are very similar, particularly for the relative formation energies in Table IV which all
TABLE IV. Energy change for the exchange of a substitutional carbon atom in host $a$ with an atom of the type it substitutes for from host $b$ : $\left[E(b)_{\text {defect }}-E(b)_{\text {ideal }}\right]-\left[E(a)_{\text {defect }}-E(a)_{\text {ideal }}\right]$.

\begin{tabular}{|c|c|c|c|}
\hline \multicolumn{4}{|c|}{$\mathrm{C}_{M}(a) \rightarrow \mathrm{C}_{M}(b)(\mathrm{eV})$} \\
\hline$M$ & $M \mathrm{P} \rightarrow M$ As & $M \mathrm{As} \rightarrow M \mathrm{Sb}$ & $M \mathrm{P} \rightarrow M \mathrm{Sb}$ \\
\hline \multicolumn{4}{|c|}{ 64-atom supercell } \\
\hline $\mathrm{Al}$ & 0.00 & -0.73 & -0.73 \\
\hline $\mathrm{Ga}$ & -0.04 & -0.53 & -0.57 \\
\hline In & -0.08 & -0.52 & -0.60 \\
\hline \multicolumn{4}{|c|}{ 216-atom supercell } \\
\hline $\mathrm{Al}$ & 0.03 & -0.74 & -0.72 \\
\hline $\mathrm{Ga}$ & -0.04 & -0.57 & -0.61 \\
\hline In & -0.08 & -0.53 & -0.61 \\
\hline \multicolumn{4}{|c|}{$\mathrm{C}_{X}(a) \rightarrow \mathrm{C}_{X}(b)(\mathrm{eV})$} \\
\hline$X$ & $\mathrm{Al} X \rightarrow \operatorname{Ga} X$ & $\operatorname{Ga} X \rightarrow \operatorname{In} X$ & Al $X \rightarrow \operatorname{In} X$ \\
\hline \multicolumn{4}{|c|}{ 64-atom supercell } \\
\hline$P$ & -0.08 & 0.51 & 0.43 \\
\hline As & 0.03 & 0.55 & 0.58 \\
\hline $\mathrm{Sb}$ & 0.31 & 0.46 & 0.77 \\
\hline \multicolumn{4}{|c|}{ 216-atom supercell } \\
\hline $\mathrm{P}$ & -0.14 & 0.54 & 0.40 \\
\hline As & -0.04 & 0.59 & 0.55 \\
\hline $\mathrm{Sb}$ & 0.24 & 0.52 & 0.76 \\
\hline
\end{tabular}

agree to $0.08 \mathrm{eV}$ or better. The main difference is in the formation energies relative to an arbitrary chemical potential (Table III). These are all slightly higher in the 216-atom supercells than in the 64-atom ones. For $\mathrm{C}_{M}$ and $\mathrm{C}_{\mathrm{As}}$ in AlAs, GaAs, and InAs, the amounts by which they are larger in the bigger supercells are within $0.05 \mathrm{eV}$ of the corresponding figures for the energy of relaxation of the lattice parameter given in Table II. Hence, the obvious conclusion is that the unrelaxed strain in the 64-atom supercells accounts for nearly all the energy differences.

The story is similar for structural parameters. Figure 1 defines the bond lengths and angles in the neighborhood of a

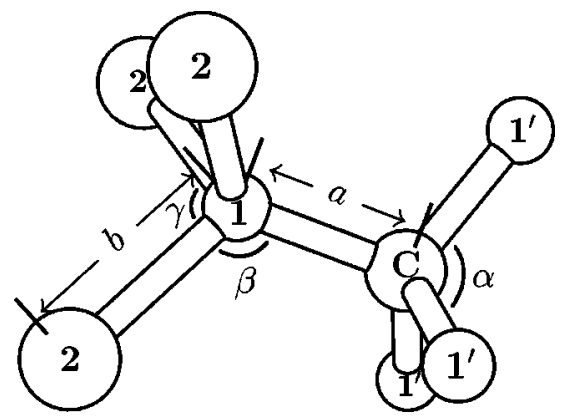

FIG. 1. Structural parameters for a substitutional carbon atom defect with perfect $T_{d}$ symmetry. The nearest neighbor bond length is $a$ and second nearest neighbor is $b$. All the bond angles $\alpha$ at the carbon atom $\mathrm{C}$ are fixed at $109^{\circ} 30^{\prime}$; all atoms marked 1 or $1^{\prime}$ are equivalent. There are two unique bond angles at atom 1: between $\mathrm{C}$ and any atom labeled $2(\beta)$, and between any pair of atoms marked $2(\gamma)$. 
TABLE V. Calculated bond lengths ( $\mathrm{A})$ for the 216-atom supercells. See Fig. 1.

\begin{tabular}{lccccc}
\hline \hline$M X$ & $\mathrm{C}_{M}-X$ & $\mathrm{C}_{X}-M$ & $X-M\left(\mathrm{C}_{M}\right)$ & $M-X\left(\mathrm{C}_{X}\right)$ & $X-M$ (ideal) \\
\hline $\mathrm{AlP}$ & 1.9933 & 1.9807 & 2.4049 & 2.4120 & 2.3520 \\
$\mathrm{AlAs}$ & 2.1048 & 1.9912 & 2.4809 & 2.5003 & 2.4334 \\
$\mathrm{AlSb}$ & 2.2872 & 2.0135 & 2.6807 & 2.7290 & 2.6373 \\
$\mathrm{GaP}$ & 1.9969 & 1.9522 & 2.3474 & 2.3620 & 2.3016 \\
$\mathrm{GaAs}$ & 2.1016 & 1.9634 & 2.4352 & 2.4569 & 2.3876 \\
$\mathrm{GaSb}$ & 2.2854 & 1.9883 & 2.6232 & 2.6789 & 2.5812 \\
$\mathrm{InP}$ & 2.0062 & 2.1045 & 2.5257 & 2.5117 & 2.4573 \\
$\mathrm{InAs}$ & 2.1103 & 2.1155 & 2.6068 & 2.5978 & 2.5382 \\
$\mathrm{InSb}$ & 2.2981 & 2.1373 & 2.7856 & 2.8105 & 2.7272 \\
\hline \hline
\end{tabular}

single substitutional carbon atom, and the calculated values for the 216-atom supercells are given in Tables V and VI. Figure 2 is a convenient graphical representation of the bond lengths. A detailed analysis of all the calculated parameters shows that both the nearest and second nearest neighbor bond lengths in all cases are very slightly smaller in the larger supercell. The average amount for the nearest neighbor bonds is $-0.41 \%$, and for second nearest neighbor bonds is $-0.26 \%$. Beyond the second nearest neighbors the atom positions differ very little from the ideal lattice. We can, therefore, be reasonably confident that the properties calculated here for the single substitutional carbon atom are well described by our models.

Trends in the formation energies with elemental composition relative to one another are small, yet significantly larger than the level of sensitivity demonstrated by the method $(\sim 0.05 \mathrm{eV})$. When carbon replaces $\mathrm{Al}, \mathrm{Ga}$, or In, the formation energy is nearly the same in phosphides and arsenides, but is clearly lower in antimonides by several tenths of an $\mathrm{eV}$. In other words, the best chance of finding carbon donors is in antimonides. This difference in energy is greatest for $\mathrm{AlSb}: \mathrm{C}_{\mathrm{Al}}$ compared with $\mathrm{AlAs}: \mathrm{C}_{\mathrm{Al}}$ and $\mathrm{AlP}: \mathrm{C}_{\mathrm{Al}}$. There is perhaps a slight indication that as the metal changes from $\mathrm{Al}$ to $\mathrm{Ga}$ to In the balance in energy changes from favoring the phosphides to the arsenides, although this is at the limit of what could be considered reliable. When carbon replaces $\mathrm{P}$, As, or $\mathrm{Sb}$, the trend in formation energy with respect to atomic number of the substituted atom is now the other way around, so that $\mathrm{C}$ atoms would prefer to be in $\mathrm{Al}$ or Ga compounds than in In ones. The largest energy difference is between $\mathrm{AlSb}: \mathrm{C}_{\mathrm{Sb}}$ and $\mathrm{InSb}: \mathrm{C}_{\mathrm{Sb}}$. There is also a clearer differential between $\mathrm{Al}$ and $\mathrm{Ga}$ compounds than between $\mathrm{P}$ and As compounds. The balance switches from the $\mathrm{Al}$ compound being favored to the $\mathrm{Ga}$ one as the atom that $\mathrm{C}$ replaces is changed from $\mathrm{P}$ to $\mathrm{As}$ to $\mathrm{Sb}$, with $\mathrm{AlAs}: \mathrm{C}_{\mathrm{As}}$ and $\mathrm{GaP}: \mathrm{C}_{\mathrm{As}}$ having essentially equal formation energies.

The predicted behavior of some of these combinations is therefore in good agreement with the experimental observations reported by Abernathy. For example, GaP, AlAs, and GaAs are strongly $p$ type when doped with carbon. In other cases the opposite appears to happen. This has some similarities with the situation found by Weyers and Shiraishi, except that now more information is available to provide possible explanations. In the case of InSb doped with carbon,
TABLE VI. Calculated bond angles for the 216-atom supercells. See Fig. 1.

\begin{tabular}{lcccc}
\hline \hline$M X$ & $\beta\left(\mathrm{C}_{M}\right)$ & $\beta\left(\mathrm{C}_{X}\right)$ & $\gamma\left(\mathrm{C}_{M}\right)$ & $\gamma\left(\mathrm{C}_{X}\right)$ \\
\hline AlP & $115^{\circ} 42^{\prime}$ & $116^{\circ} 57^{\prime}$ & $102^{\circ} 35^{\prime}$ & $101^{\circ} 04^{\prime}$ \\
AlAs & $115^{\circ} 01^{\prime}$ & $117^{\circ} 57^{\prime}$ & $103^{\circ} 24^{\prime}$ & $99^{\circ} 48^{\prime}$ \\
AlSb & $114^{\circ} 57^{\prime}$ & $120^{\circ} 11^{\prime}$ & $103^{\circ} 29^{\prime}$ & $96^{\circ} 57^{\prime}$ \\
$\mathrm{GaP}$ & $114^{\circ} 55^{\prime}$ & $116^{\circ} 34^{\prime}$ & $103^{\circ} 31^{\prime}$ & $101^{\circ} 32^{\prime}$ \\
$\mathrm{GaAs}$ & $114^{\circ} 20^{\prime}$ & $117^{\circ} 40^{\prime}$ & $104^{\circ} 12^{\prime}$ & $100^{\circ} 11^{\prime}$ \\
$\mathrm{GaSb}$ & $114^{\circ} 06^{\prime}$ & $119^{\circ} 45^{\prime}$ & $104^{\circ} 29^{\prime}$ & $97^{\circ} 31^{\prime}$ \\
$\mathrm{InP}$ & $117^{\circ} 09^{\prime}$ & $116^{\circ} 18^{\prime}$ & $100^{\circ} 50^{\prime}$ & $101^{\circ} 52^{\prime}$ \\
$\mathrm{InAs}$ & $116^{\circ} 28^{\prime}$ & $117^{\circ} 16^{\prime}$ & $101^{\circ} 40^{\prime}$ & $100^{\circ} 40^{\prime}$ \\
InSb & $115^{\circ} 57^{\prime}$ & $119^{\circ} 22^{\prime}$ & $102^{\circ} 18^{\prime}$ & $98^{\circ} 01^{\prime}$ \\
\hline \hline
\end{tabular}

hole concentrations as high as $\sim 5 \times 10^{20} \mathrm{~cm}^{-3}$ are reported, although the lattice site of the incorporated carbon (presumably $\mathrm{C}_{\mathrm{Sb}}$ ) is not identified. ${ }^{15}$ This situation is unusual in that it does not follow the general trends, both predicted and observed, for the other In and Sb compounds. First, of the three antimonides examined here, this is the least favored for $\mathrm{C}$ to replace $\mathrm{Sb}$. Moreover, out of the three In compounds, $\mathrm{C}_{\mathrm{In}}$ has by far the lowest energy in InSb. Carbon in InP, as mentioned previously, is observed to occupy only the $\mathrm{P}$ site, yet the material is semi-insulating, probably due to compensating defects dominating the electronic properties. The behavior of InAs:C depends on the growth precursors, but it too is always observed to be compensated..$^{16} \mathrm{Ab}$ initio pseudopotential calculations by Lee and Chang predict that arsenicantisite defects $\mathrm{As}_{\mathrm{In}}$ have a lower formation energy than ei-

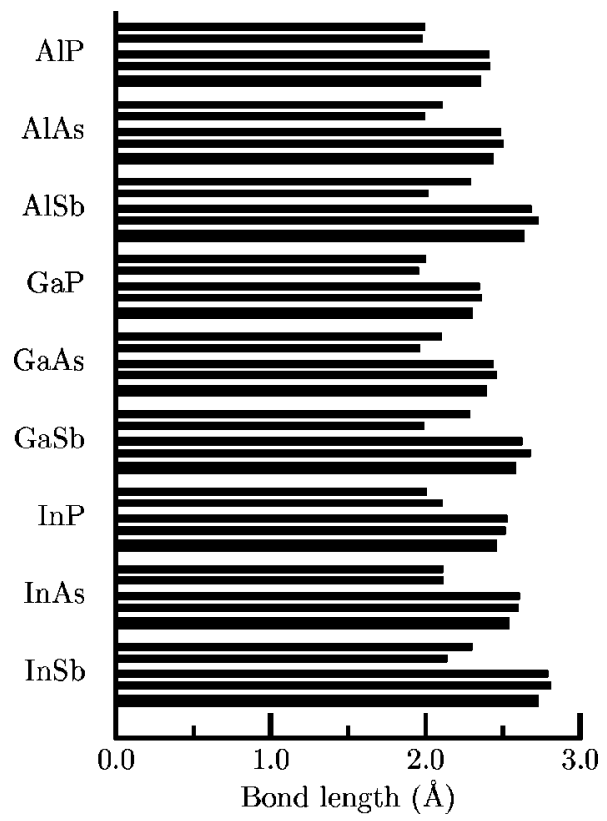

FIG. 2. Calculated lengths of nearest and second nearest neighbor bonds to a single substitutional carbon atom in a 216-atom supercell, and in the pure, ideal crystals. For each compound, the ordering from the top is nearest neighbors (first pair of lines) $\mathrm{C}_{M^{-}} X$, and $\mathrm{C}_{X}-M$; second nearest neighbors (second pair of lines) $X-M$, and $M-X$; ideal (thick lines). 
ther $\mathrm{C}_{\mathrm{As}}$ or $\mathrm{C}_{\mathrm{In}}$ (which has an even higher formation energy) in InAs. ${ }^{17}$ They suggest that the relatively small size of carbon atoms accounts for the comparatively large formation energy for substitutional carbon defects. Thus, if InSb followed the general trends, it would be expected to be compensated, and might even be $n$ type in the right circumstances.

Returning to the structural parameters, the general picture is similar in every case. The bonds that carbon-a relatively small atom-forms with its four nearest neighbors are shorter than those for the ideal host crystal. When carbon replaces $\mathrm{Al}, \mathrm{Ga}$, or $\mathrm{In}$, the $\mathrm{C}$ atom's bond lengths are 88$82 \%$ of the host's, and when P, As, or Sb are substituted by $\mathrm{C}$ the range of values is $86-76 \%$ of the bond length for the host. The bond lengths and angles of the second nearest neighboring atom to a substitutional carbon atom are then forced to stretch slightly to accommodate the strain. A figure of about $102 \%$ is typical for the ratio of the second neighbor bond length to that for the ideal crystal. This accounts for nearly all the distortions: the atomic positions of more distant neighbors are very close to those for an ideal lattice.

\section{SUMMARY AND CONCLUSIONS}

The main aim of this study was to search for trends in the energetics of carbon doping of III-V semiconductors. This objective has largely been achieved. Using the supercell version of the AIMPRO local-density-functional theoretical method, basis sets were first constructed and tested for the atomic species under consideration. Satisfactory results were acheived in most cases, with the exception of $\mathrm{GaN}$ and InN, which were not judged to be a success for reasons discussed in Sec. III A. We then focused on all the possible combinations of $\mathrm{Al}, \mathrm{Ga}$, and In with $\mathrm{P}, \mathrm{As}$, and $\mathrm{Sb}$ containing a single substitutional carbon atom in supercells of 64 and 216 atoms. A subset of the calculations on the arsenides showed that the lattice parameter $a_{0}$ contracted by an amount in good agreement with Vegard's law in 64-atom supercells, and that the 216-atom supercells model the structural parameters and energetics of a single substitutional carbon atom well. Over the whole set of compound semiconductors, two main trends were found. If carbon is placed on the site of one of the metal atoms, the balance of the formation energies favors the antimonides over the phosphides and arsenides. If instead $\mathrm{C}$ replaces $\mathrm{P}$, As, or $\mathrm{Sb}$, the energetics favor the $\mathrm{Al}$ and $\mathrm{Ga}$ compounds over the In ones. Therefore, the chances of carbon acting as an $n$-type dopant among the set of compounds modeled here is greatest in InSb, and the probability of carbon showing $p$-type behavior is highest for GaP, AlAs, and GaAs. The observed electronic properties are sometimes found to follow the expected trends; however, this is not always the case. Compensating defects and the details of the processes by which different crystals are grown can be the dominant influences. The stuctural parameters in all cases simply reflect the fact that carbon atoms have a relatively small size. The resulting strain is almost entirely accommodated by an inward distortion of the positions of the four nearest neighbors to the $\mathrm{C}$ atom.

Finally, the results of this work imply that there may be special consequences of using carbon as a dopant in compound semiconductors consisting of more than two elemental species, or in heterojunction devices including those with graded layers. In these circumstances the competition for which site carbon preferentially occupies becomes more complex. For example, in a compound containing both $\mathrm{Ga}$ and $\mathrm{In}$, it is likely that the concentration of any carbon acceptors present will be locally higher in regions where there is an excess of Ga. This may or may not be what is desired by the designer of a device.

\section{ACKNOWLEDGMENTS}

The Engineering and Physical Sciences Research Council (EPSRC), U.K., is thanked for its financial support at Exeter University (Contract No. GR/L34457). S.Ö. would like to thank TFR in Sweden for financial support. The authors would also like to express their thanks in particular to J. Coutinho, C. J. Fall, and M. O. Kaukonen for helpful discussions and providing the results of their calculations.
*Email address: C.D.Latham@ex.ac.uk

${ }^{1}$ C. R. Abernathy, in State-of-the-Art Program on Compound Semiconductors XXIV, edited by F. Ren, S. J. Pearton, and W. Pletschen (The Electrochemical Society, Pennington, NJ, 1996), pp. $1-18$.

${ }^{2}$ H. Ito and T. Ishibashi, Jpn. J. Appl. Phys., Part 2 30, L944 (1991).

${ }^{3}$ R. C. Newman, B. R. Davidson, R. S. Leigh, M. J. L. Sangster, and C. C. Button, Physica B 273-274, 827 (1999).

${ }^{4}$ B. R. Davidson, R. C. Newman, and C. C. Button, Phys. Rev. B 58, 15609 (1998).

${ }^{5}$ J. Wagner, R. C. Newman, B. R. Davidson, S. P. Westwater, T. J. Bullough, T. B. Joyce, C. D. Latham, R. Jones, and S. Öberg, Phys. Rev. Lett. 78, 74 (1997).

${ }^{6}$ C. D. Latham, R. Jones, B. R. Davidson, R. C. Newman, C. C.
Button, P. R. Briddon, and S. Öberg, Phys. Status Solidi B 210, 869 (1998).

${ }^{7}$ B. R. Davidson, R. C. Newman, C. D. Latham, R. Jones, J. Wagner, C. C. Button, and P. R. Briddon, Phys. Rev. B 60, 5447 (1999).

${ }^{8}$ R. C. Newman (private communication).

${ }^{9}$ M. Weyers and K. Shiraishi, Jpn. J. Appl. Phys., Part 1 31, 2483 (1992).

${ }^{10}$ J. Coutinho, R. Jones, P. R. Briddon, and S. Öberg Phys. Rev. B 62, 10824 (2000).

${ }^{11}$ P. R. Briddon, Ph.D. thesis, Department of Physics, University of Exeter, United Kingdom, 1990.

${ }^{12}$ J. P. Perdew and Y. Wang, Phys. Rev. B 45, 13244 (1992).

${ }^{13}$ G. B. Bachelet, D. R. Hamann, and M. Schlüter, Phys. Rev. B 26, 4199 (1982). 
${ }^{14}$ H. J. Monkhorst and J. D. Pack, Phys. Rev. B 13, 5188 (1976).

${ }^{15}$ W. V. Schoenfeld, M. J. Antonell, and C. R. Abernathy, Appl. Phys. Lett. 72, 1235 (1998).

${ }^{16}$ W. V. Schoenfeld, M. J. Antonell, and C. R. Abernathy, J. Cryst. Growth 188, 50 (1998).
${ }^{17}$ S. G. Lee and K. J. Chang, Phys. Rev. B 53, 9784 (1996).

${ }^{18} \mathrm{~J}$. Singh, Physics of Semiconductors and Their Heterostructures (McGraw-Hill, New York, 1993).

${ }^{19}$ CRC Handbook of Chemistry and Physics, 77th ed., edited by D. R. Lide (CRC Press, Boca Raton, FL, 1996). 